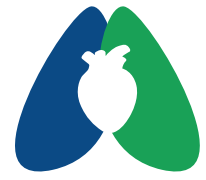

ASSOBRAFIR C I Ê N C I A

\title{
O impacto da reabilitação cardiovascular sobre a qualidade de vida de pacientes portadores de doença arterial coronariana
}

\author{
The impact of cardiovascular rehabilitation on the quality of life in \\ patients with coronary heart disease
}

\author{
Roberta Castro Lopes $^{1}$ (1); Daniel Meneguzzi ${ }^{2}$ (1); Costantino Roberto Costantini ${ }^{1}$ (1); \\ Costantino Costantini Ortiz' (1D; Alexandre Rodrigues dos Santos Kopka1 (D); \\ Ana Carolina Brandt de Macedo ${ }^{3}$ (D); Rafael Michel de Macedo ${ }^{1}$ (D) \\ ${ }^{1}$ Hospital Cardiológico Costantini, Curitiba, PR, Brasil \\ ${ }^{2}$ Curso de Fisioterapia, Centro Universitário Campus de Andrade (UNIANDRADE), Curitiba, PR, Brasil \\ ${ }^{3}$ Departamento de Prevenção e Reabilitação em Fisioterapia, Curitiba, PR, Brasil
}

Como citar: Lopes RC, Meneguzzi D, Constantini CR, Constantini CO, Kopka ARS, de Macedo ACB, et al. O impacto da reabilitação cardiovascular sobre a qualidade de vida de pacientes portadores de doença arterial coronariana. ASSOBRAFIR Ciênc. 2021;12:e42694. https:// doi.org/10.47066/2177-9333.AC.2020.0028

\begin{abstract}
Resumo
Introdução: A doença arterial coronariana (DAC) pode causar acometimento funcional, dependência física e risco de morte, prejudicando a qualidade de vida. A reabilitação cardiovascular consiste no conjunto de práticas que visam a melhora das condições físicas, mentais e sociais do indivíduo coronariopata. Objetivo: Avaliar o impacto do programa de reabilitação cardiovascular com ênfase no exercício perante a qualidade de vida de pacientes portadores de DAC. Métodos: Trata- se de um estudo observacional retrospectivo. Foram selecionados de forma aleatória 30 prontuários de pacientes portadores de DAC que completaram a fase II do programa de treinamento supervisionado da Academia do Coração do Hospital Cardiológico Costantini, entre os anos de 2012 e 2017. Foram avaliadas a qualidade de vida por meio do questionário SF-36, a composição corporal e a aptidão cardiorrespiratória e física por meio do teste ergoespirométrico e de resistência máxima (RM) respectivamente. Resultados: Foram encontradas diferenças significativas nos domínios aspectos físicos $(p=0,01)$, estado geral de saúde $(p=0,00)$, vitalidade $(p=0,00)$ e saúde mental $(p=0,00)$, aumento da massa magra $(p=0,00)$ e melhora no $\mathrm{VO}_{2}$ limiar anaeróbio, $\mathrm{VO}_{2}$ pico e força muscular (RM) $(p<0,05)$. Conclusão: O programa de treinamento físico contribuiu para a melhora da qualidade de vida dos pacientes portadores de DAC e também na aptidão cardiorrespiratória e física.
\end{abstract}

Palavras-chave: Reabilitação; Doenças Cardiovasculares; Qualidade de Vida.

\begin{abstract}
Background: Coronary artery disease (CAD) can cause functional impairment, physical dependence and risk of death, impairing quality of life. Cardiovascular rehabilitation consists of a set of practices aimed at improving the physical, mental and social conditions of the coronary patient. Aim: To evaluate the impact of cardiovascular rehabilitation program with emphasis on exercise in the quality of life of patients with CAD. Methods: This is a retrospective analytical study. It was randomly selected 30 charts of patients with CAD who completed phase II of the supervised training program of the Heart Academy of Costantini Cardiology Hospital between 2012 and 2017. Quality of life was assessed by questionnaire SF-36 and cardiorespiratory and physical fitness by ergospirometric and maximum resistance test (MR), respectively. Results: There was a significant difference physical aspects $(p=0.01)$, general health status $(p=0.00)$, vitality $(p=0.00)$ and mental health $(p=0.00)$, increase in lean mass $(p=0.00)$, improvement $\mathrm{VO}_{2}$ anaerobic threshold and peak $\mathrm{VO}_{2}$ and muscle strength (MRI) $(p=0.00)$. Conclusion: The physical training program contributed to improve the quality of life of patients with CAD, and also in cardiorespiratory fitness and physical fitness. Keywords: Rehabilitation; Cardiovascular Diseases; Quality of Life.
\end{abstract}

Estudo realizado em: Hospital Cardiológico Costantini, Curitiba, PR, Brasil. Aprovação ética: CAAE: 69444717.3.0000.5218 do Centro Universitário Campos de Andrade, n061877/2017.

*Autor correspondente:

Rafael Michel de Macedo. E-mail:

rafael.macedo@hospitalcostantini.com.br

Este é um artigo publicado em acesso aberto (Open Access) e distribuído sob a licença Creative Commons Attribution NonComercial ShareAlike License, que permite uso, distribuição e reprodução em qualquer meio, sem restrições desde que sem fins comerciais e que o trabalho original seja corretamente citado e de forma que não indique endosso ao trabalho feito. Adicionalmente, qualquer trabalho derivado deverá ser publicado sob a mesma licença. 


\section{INTRODUÇÃO}

As doenças cardiovasculares (DCV) constituem aproximadamente $48 \%$ da causas de mortes no mundo e $30 \%$ no Brasil'1. Segundo a Organização mundial da saúde (OMS), estima-se que as DCVs foram a causa de morte de 17,8 milhões de pessoas em 2017, o que representou $31 \%$ das mortes a nível global, sendo a doença arterial coronariana (DAC) a de maior frequência e uma das principais doenças crônicas não transmissíveis (DCNTs)².

As consequências da DAC incluem acometimento funcional, dependência física e risco de morte, prejudicando a qualidade de vida (QV) dessa população ${ }^{3}$. O acometimento funcional é causado, principalmente, pelo sedentarismo que é caracterizado como uma "doença hipocinética" afetando diretamente o sistema cardiorrespiratório e locomotor e sua cronicidade pode provocar a hipóxia miocárdica. Sendo assim, o combate ao sedentarismo passa a ser objetivo primário do tratamento preventivo e reabilitativo da $\mathrm{DAC}^{4}$.

Faz-se importante ressaltar que o tratamento da DAC divide-se em clínico e cirúrgico. O tratamento cirúrgico pode ser realizado de maneira percutânea, por meio de angioplastia transluminal coronariana (ATC) e ou por cirurgia de revascularização do miocárdio (CRM). Já o tratamento clínico, divide-se em farmacológico e não farmacológico, sendo o exercício físico parte fundamental do grupo de medidas não farmacológicas ${ }^{4}$.

Segundo a $\mathrm{OMS}^{5}$, a reabilitação cardíaca (RC) consiste no conjunto de práticas necessárias para a reinclusão de portadores de doenças cardíacas na comunidade, com melhores condições físicas, mentais e sociais. Desta forma, os programas de RC têm sido desenvolvidos com base no treinamento físico pela prescrição de exercícios baseados nas necessidades de cada pessoa, juntamente com ações educativas para mudança no estilo de vida, como por exemplo o sedentarismo ${ }^{6}$. Ela é divida em 4 fases: Fase I ou hospitalar, Fase II ou ambulatorial, Fase III ou transição e Fase IV ou não supervisionada?.

A fase II é a fase supervisionada de convalescença, com duração de aproximadamente três meses. Tem como objetivo principal modificações de fatores de risco, melhora da capacidade funcional, qualidade de vida e recuperação de autoconfiança, no qual, são prescritos exercícios aeróbicos, de resistência e flexibilidade com progressão individualizada7.

Além das limitações físicas, indivíduos portadores de doença crônica, como a DAC, deparam-se com maior dificuldade em desempenhar suas funções psíquicas e sociais, afetando ainda mais sua QV2. Faz-se importante ressaltar que a QV está diretamente relacionada com a evolução clínica favorável do paciente e pode ser medida de maneira quantitativa, o que permite que os profissionais da saúde avaliem o impacto da sua ação sob o tratamento da doença ${ }^{8}$.
Sampaio et al. ${ }^{9}$ avaliaram a QV de 114 pacientes pós angioplastia ou revascularização do miocárdio por meio do questionário de qualidade de vida Short-form 36 (SF-36). Verificaram que o maior comprometimento antes da cirurgia eram nos aspectos físicos, capacidade funcional e emocional e que após 3 meses houve melhora desses domínios porém sem melhora no social e emocional. Após 6 meses não encontraram melhora nos domínios físico e emocional. Esses autores não descrevem se os pacientes foram submetidos a algum programa de reabilitação.

Estudos prévios, utilizando o questionário SF-36, apontam que programas ambulatoriais de RC produzem melhora na QV de pacientes cardiopatas, repercutindo de maneira relevante na prática clínica ${ }^{10,11}$. Ainda que os trabalhos apontem essa melhora, pode-se observar que são escassos estudos investigando a influência do RC na qualidade de vida de pacientes com DAC. A revisão sistemática de Anderson et al. ${ }^{12}$ encontrou 20 estudos sobre a influência da reabilitação cardíaca na QV. Desses, 14 apontaram melhora na QV, sendo que somente 5 apresentaram aumento de mais de $50 \%$. Esses autores apontam que os estudos que avaliam a QV são heterogêneos em relação a amostra e instrumento utilizado e que são necessárias mais pesquisas para elucidar o tema.

Portanto, o objetivo primário deste trabalho foi avaliar o impacto do programa de RC com ênfase no exercício perante a QV de pacientes portadores de DAC e como objetivo secundário avaliar o consumo máximo de oxigênio $\left(\mathrm{VO}_{2}\right), \mathrm{VO}_{2}$ pico e força muscular.

\section{MÉTODOS}

\section{Tipo de estudo}

Trata- se de um estudo observacional retrospectivo, realizado após a aprovação do comitê de ética CAAE: 69444717.3.0000.5218 do Centro Universitário Campos de Andrade, número 061877/2017. Todos os indivíduos envolvidos assinaram o Termo de Consentimento Livre e Esclarecido.

\section{População estudada}

Foram selecionados de forma aleatória prontuários de pacientes portadores de DAC que completaram a fase II do programa de treinamento supervisionado da Academia do Coração do Hospital Cardiológico Costantini (HCC) entre os anos de 2012 e 2017.

\section{Critérios de inclusão}

Foram incluídos no estudo os prontuários de pacientes que apresentavam preenchidos os questionários de qualidade de vida SF-36 pré e pós tratamento, armazenados de forma digital dentro do sistema de prontuário eletrônico TASY $(n=30)$. 
Critérios de exclusão

Foram excluídos os pacientes que apesar de preencherem o questionário não compareceram a no mínimo 65\% das sessões do programa de reabilitação.

Metodologia de Avaliação e Reavaliação do Programa de reabilitação Cardiovascular

Todos os pacientes incluídos neste estudo foram encaminhados pelos seus médicos para iniciar o programa de treinamento supervisionado da Academia do Coração com teste cardiopulmonar (TCP) já realizado.

$\mathrm{Na}$ avaliação inicial com o fisioterapeuta, os pacientes foram submetidos a uma avaliação de composição corporal pelo protocolo de Faulkner (pregas e circunferências) ${ }^{8}$, sendo utilizado para tal adipômetro da marca CESCORF (Porto Alegre, Brasil) e uma fita métrica. Foram realizadas 3 medidas para cada circunferência sendo considerada a média das três tentativas para as seguintes pregas: tricipital, subescapular, suprailíaca e abdominal. Além disso, foram medidas as seguintes circunferências: Panturrilhas, coxa, abdôme e bíceps relaxado. Os cálculos de $\%$ de gordura e de massa magra foram realizados em planilha de Excel fornecida pela CESCORF.

Todos os dados da avaliação foram alimentados no sistema próprio da academia HEALTH SYSTEM PROGRAM da marca PHILIPS (Brasil), trata se de um sistema incorporado ao prontuário eletrônico (sistema TASY do hospital), onde foram armazenados todos os dados.

Este modelo de avaliação foi repetido após o cumprimento da fase II do programa de reabilitação a fim de comparar os resultados obtidos.

\section{Teste cardiopulmonar}

Os testes foram executados por médicos, em ambiente preparado dentro do Hospital. O protocolo utilizado para avaliação foi o de rampa, com variação de velocidade definida pelo executor de acordo com as características físicas de cada paciente. O conjunto ergoespirométrico foi composto: pelo analisador de gases METALYZER da CORTEX (Brasil), uma esteira INBRAMED (Porto Alegre, Brasil) com velocidade máxima de $22 \mathrm{~km} / \mathrm{h}$, o sistema da Micromed de avaliação cardiovascular. A monitorização eletrocardiográfica foi constante, sendo utilizados os eletrodos (3M) e o um esfigmomanômetro e estetoscópio marca BD para aferição da pressão arterial a cada 2 min e em fase de recuperação pós teste.

Para determinação dos limiares ventilatório foi utilizado o método computadorizado V-Slope por meio de uma análise visual através da intersecção entre os equivalentes ventilatórios de oxigênio $\left(\mathrm{VE} / \mathrm{VO}_{2}\right)$ e dióxido de carbono $\left(\mathrm{VE} / \mathrm{VCO}_{2}\right)$ chamado de turning point para determinar o primeiro limiar ventilatório (limiar anaeróbio). Já para o segundo limiar ventilatório ou ponto de compensação ventilatória a descontinuidade da relação $\mathrm{VE} / \mathrm{VCO}_{2}$. O VO $\mathrm{V}_{2}$ pico ou consumo máximo de oxigênio foi determinado pela descontinuidade da elevação ou platô do $\mathrm{VO}_{2}$ mesmo com elevação da carga de trabalho, e para determinar o valor predito foi utilizada equação em relação ao peso $\left(\mathrm{ml}^{\mathrm{kg}}{ }^{-1} \cdot \mathrm{min}^{-1}\right)$.

\section{Teste de uma repetição máxima}

Trata-se de um teste neuromuscular de 1 repetição máxima (1RM) realizado com carga progressiva sendo a carga máxima definida como aquela abaixo do movimento não executado ou executado com compensações. O teste foi iniciado com um aquecimento de 3 a 5 repetições submáximas para o indivíduo familiarizar-se com o movimento, desta forma o paciente teve uma tentativa para cada grupo muscular. Foi selecionada uma carga inicial para realizar o teste e se o indivíduo conseguisse executar o movimento aumenta-se a carga de 5 em $5 \mathrm{Kg}$ na máquina. Os intervalos entre as séries foram estabelecidos de acordo com a recuperação de cada paciente. Foram testados no dia da avaliação 8 grupos musculares em equipamentos de musculação da marca MOVEMENT (São Paulo, Brasil) (Cadeira Adutora, Cadeira Abdutora, Cadeira Extensora, Cadeira Flexora, Voador, Polia Alta Costa, Tríceps Cross, Banco Scott) $)^{13}$.

\section{Questionário de Qualidade de Vida SF-36}

Trata-se de um questionário auto-admistrável, validado para a língua portuguesa ${ }^{14}$ composto por 36 questões divididas em 8 categorias referentes a QV: capacidade funcional (CF) (dez itens), aspectos físicos (AF) (quatro itens), dor (dois itens), estado geral da saúde (EGS) (cinco itens), vitalidade (quatro itens), aspectos sociais (AS) (dois itens), aspectos emocionais (AE) (três itens), saúde mental (SM) (cinco itens) e uma questão ( $\left.\mathrm{n}^{\circ} .2\right)$ comparativa sobre a percepção atual da saúde (PAS) e há um ano.

Para cada domínio do questionário é estabelecido um escore de 0 a 100, sendo zero o pior e 100 o melhor. A partir das respostas obtidas por cada candidato, as perguntas são dividas em cada aspecto de qualidade de vida segundo um valor de referência chamado Raw Scale Os escores foram obtidos por meio de uma fórmula específica ${ }^{14}$, onde os valores para o "Limite Inferior" e "Variação (Score Range)" são fixos dados pelo Raw Scale. O questionário foi aplicado antes do início do programa de reabilitação e após seu término (36 sessões).

Ao término do teste cardiopulmonar todos os pacientes receberam o questionário de qualidade de vida SF$36^{14}$ impresso o qual foi auto preenchido e entregue para o fisioterapeuta na avaliação inicial. Todos os dados foram preenchidos no sistema TASY pela técnica de enfermagem da esgoespirometria e foram armazenados na forma digital.

\section{Protocolo de treinamento}

Os pacientes foram submetidos ao programa de exercícios durante 36 sessões de treino supervisionado, $3 \mathrm{X}$ 
por semana, contendo exercícios aeróbios (EA) e exercícios resistidos (ER).

Os exercícios aeróbicos foram realizados em esteira elétrica, marca MOVEMENT, modelos RT250, LX160 e $L X 150$, e os resistidos, realizados com caneleiras, halteres e em aparelho de musculação (ESTAÇÃO MEGA MOVEMENT II). Em 24 sessões foram realizados treino em esteira (TE) e resistido de membros inferiores (MMII), enquanto em 12 , TE e resistido de membros superiores (MMSS). A cada duas sessões de TE+MMII foi realizada uma de TE+MMSS.

Os exercícios que compuseram as sessões de treinamento de MMII com caneleiras foram: extensão de joelhos e flexão do quadril (sentado). Na posição em pé: flexão de joelhos, abdução e adução de quadril, plantiflexão de tornozelos e flexão de quadril associada a de joelho. No aparelho: extensão e flexão dos joelhos. Já, os que compuseram as sessões de MMSS com halteres foram: em pé: flexão de cotovelos, extensão de cotovelos, abdução de ombro, adução de escápulas, flexão anterior do ombro, exercício pendular para decoaptação articular do ombro; sentado: adução de escápulas. No aparelho: supino reto, bíceps, tríceps e polia. O que determinou a utilização de caneleiras/halteres ou aparelho foi o resultado do teste de 1RM. A intensidade do treinamento resistido variou entre $30-50 \%$ das cargas obtidas neste teste. Foram realizadas 3 séries de 15 repetições de cada exercício.

A intensidade do exercício aeróbico em esteira elétrica para os grupos foi definida a partir do resultado do TCP laudado pelo médico contendo as especificações dos limiares ventilatórios. A frequência cardíaca (FC) correspondente ao limiar ventilatório 1 (LV1) foi definida como limite inferior de treinamento (FC1), já a FC correspondente ao limiar ventilatório 2 (LV2) como limite superior (FC2). O intervalo entre FC1-FC2 corresponde a intensidade ideal de treinamento para cada paciente, sendo chamada de zona alvo $(Z A)^{3}$.

Os pacientes começaram o programa de treinamento aeróbico com 25 minutos de atividade, sendo $5 \mathrm{~min}$ de aquecimento, $15 \mathrm{~min}$ de treino em ZA, e $5 \mathrm{~min}$ finais de desaquecimento. A fase de aquecimento e desaquecimento foi mantida com $5 \mathrm{~min}$ cada durante todas as 36 sessões. A cada 3 sessões foi incrementado $5 \mathrm{~min}$ de treinamento dentro da ZA. A partir da $10^{a}$ sessão até a $36^{a}$ o tempo total de trabalho foi de $40 \mathrm{~min}$, sendo 30 destes dentro da ZA.

\section{Análise estatística}

Os dados coletados foram analisados por meio de estatística descritiva através do programa SPSS versão 20.0. A normalidade da amostra foi avaliada pelo teste de Shapiro-Wilk e a homogeneidade pelo teste de Levene. Foi realizado o teste de Wilcoxon para as variáveis não paramétricas na comparação pré e pós e para as variáveis paramétricas foi realizado o teste $t$ pareado. Também foi realizado o teste de correlação de Pearson entre os domínios do SF-36 e o teste de aptidão cardiorrespiratória e física. O estudo adotou o valor de $\mathrm{p}<0,05$ para a significância estatística.

\section{RESULTADOS}

Inicialmente foram eleitos de forma aleatória para o estudo 30 pacientes portadores de DAC que completaram a fase II do programa de reabilitação da Academia do Coração do HCC.

Destes 30 pacientes, a maioria era do sexo masculino $(22,73,8 \%)$. A média de idade foi de $58,9 \pm 11,6$ anos, sendo que $3(10 \%), 14(46,7 \%)$ realizaram angioplastia eletiva, $11(36,7 \%)$ tiveram um infarto agudo do miocárdio (IAM) seguido de angioplastia primária, e outros $2(6,75 \%)$ foram mantidos em tratamento clínico.

Os questionários e as avaliações físicas iniciais e finais do programa de reabilitação foram realizadas com tempo médio de 102 dias. A média de sessões cumpridas do programa foi de $25(69,4 \%)$ sessões de um total de 36 previstas realizadas. A Tabela 1 apresenta os resultados

Tabela 1. Resultados do Questionário SF 36 pré e pós fase II do programa de reabilitação cardiopulmonar.

\begin{tabular}{|c|c|c|c|}
\hline \multirow[t]{2}{*}{ Domínio SF 36} & $\begin{array}{c}\text { Pré } \\
(n=30)\end{array}$ & Pós (n=30) & \multirow[t]{2}{*}{ Valor de $\mathrm{p}$} \\
\hline & \multicolumn{2}{|c|}{ mi; máx; mediana; média $\pm D P$} & \\
\hline Capacidade funcional & $25 ; 100 ; 75 ; 71,7 \pm 20,4$ & $5 ; 100 ; 80 ; 76,0 \pm 15,5$ & 0,43 \\
\hline Limitações por aspectos físicos & $0 ; 100 ; 50 ; 48,2 \pm 38,2$ & $0 ; 100 ; 7569,6 \pm 38,1$ & $0,05^{\#}$ \\
\hline Dor & $20 ; 100 ; 8174,3 \pm 23,8$ & $41 ; 100 ; 8879,2 \pm 21,1$ & 0,44 \\
\hline Estado geral de saúde & $30 ; 97 ; 7271,5 \pm 17,9$ & $42 ; 100 ; 7272,2 \pm 16,3$ & $0,00 *$ \\
\hline Vitalidade & $35 ; 100 ; 67,566,0 \pm 16,1$ & $40 ; 100 ; 7571,8 \pm 15,2$ & $0,03 *$ \\
\hline Aspectos sociais & $25 ; 100 ; 7576,5 \pm 21,0$ & $50 ; 100 ; 81,580,6 \pm 18,3$ & 0,14 \\
\hline Limitações por aspectos emocionais & $0 ; 100 ; 6755,9 \pm 42,6$ & $0 ; 100 ; 10077,3 \pm 38,5$ & 0,15 \\
\hline Saúde mental & $40 ; 96 ; 8076,2 \pm 15,0$ & $48 ; 100 ; 8481,1 \pm 14,1$ & $0,00^{*}$ \\
\hline
\end{tabular}

valores descritos em mínima;máxima;mediana; média \pm desvio padrão. *valor de $\mathrm{P}<0,05$ (teste t pareado); \#valor de $\mathrm{P}<0,05$ (wilcoxon); questionário $\mathrm{SF} 36$ : Medical Outcomes Study 36 - Item Short - Form Health Survey. 
dos questionários SF 36 Preenchido pré e pós tratamento. $\mathrm{Na}$ análise de correlação foi encontrada diferença significativa com correlação forte entre o domínio limitação por aspectos físicos e o $\mathrm{VO}_{2}(p=0,00 ; r=0,49), \mathrm{VO}_{2}$ pico $(p=0,01 ; r=0,45)$ e RM $(p=0,004 ; r=0,51)$.

A Tabela 2 apresenta os dados comparativos da aptidão cardiorrespiratória, composição corporal e teste de resistência máxima.

\section{DISCUSSÃO}

Os principais achados deste estudo foram: melhora dos domínios: aspectos físicos (AF), estado geral da saúde (EGS), vitalidade e saúde mental; melhora do $\mathrm{VO}_{2}$ limiar anaeróbio, $\mathrm{VO}_{2}$ pico e força muscular.

Corroborando com nosso estudo, Saedi et al. ${ }^{15}$ avaliaram a QV de 100 pacientes com doenças cardiovasculares diversas, após 24 sessões de reabilitação cardíaca, composta, no entanto, somente por exercícios aeróbicos, verificando melhora nos domínios capacidade funcional (CF), AF, vitalidade, dor e EGS. No entanto, Villelabeitia Jaureguizar et al. ${ }^{16}$ somente encontraram melhora no EGS do SF-36 após 24 sessões (3x semana) de 40min de exercício aeróbico na bicicleta de pacientes com DAC.

Os resultados obtidos neste estudo referente ao domínio aspecto físico dentro da avaliação de qualidade de vida confirmam os achados da literatura que sugerem que programas de treinamento regulares, de intensidade moderada, melhoram a capacidade aeróbia e neuromuscular ${ }^{17}$.

Além disso, quando avaliados de forma comparativa, pacientes submetidos a tratamento cirúrgico e clínico, nota-se maior incremento na qualidade de vida pós reabilitação no primeiro grupo. Estes resultados também foram obtidos por Takiuti et al. ${ }^{18}$. Isto pode ser atribuído ao desuso neuromuscular dos pacientes submetidos a cirurgia no período pré operatório (do diagnóstico até a intervenção) e no pós operatório imediato, resultando em significativo decréscimo da capacidade funcional com sintomas percebidos.
Vidal et al. ${ }^{19}$ observaram melhora em quase todos os domínios do SF-36 (exceto aspectos emocionais) após de 3 meses de cirurgia de revascularização do miocárdio. Ressalta-se que nesse trabalho os pacientes não foram encaminhados a RC, somente foram instruídos a realizar caminhadas de no mínimo 30min, 3X semana. A tolerância ao exercício tende a ser maior em pacientes submetidos a revascularização completa do miocárdio, o que ocorre na maioria das vezes em pacientes submetidos a tratamento cirúrgico. A resolução de sintomas como a angina, por exemplo, permitem maior efeito de treinamento em decorrência da maior intensidade de exercício tolerada e por consequência melhora da qualidade de vida ${ }^{20}$. De acordo com Pocock et al. ${ }^{21}$ estes benefícios podem ser percebidos em até 3 anos pós o procedimento, diferentemente dos pacientes mantidos em tratamento clínico.

$\mathrm{O} \mathrm{VO}_{2}$ máximo reflete o nível de capacidade funcional do indivíduo, assim como o $\mathrm{VO}_{2}$ no limiar anaeróbio reflete a tolerância do paciente a realização de $\operatorname{AVDs}^{22}$. No mais, Benetti et al. ${ }^{23}$ demonstraram que pacientes pós IAM melhoraram os aspectos físico, social e emocional, assim como $\mathrm{V} \mathrm{VO}_{2}$ pico de forma significativa pós período de treinamento físico quando comparados aos indivíduos sedentários, que apresentaram piora em quase todos os aspectos, concluindo que quanto maior a capacidade de exercício menor o risco de morte e maior a QV.

Da mesma forma, Anzolin et al. ${ }^{24}$ compararam a melhora na QV após 45 dias de reabilitação em pacientes que realizaram cirurgia de revascularização do miocárdio, associando o exercício aeróbico ao treinamento resistido, semelhantemente a este estudo. Observaram que o aumento na força muscular e a diminuição no peso corporal tem impacto positivo na QV e também que a melhora relacionada aos aspectos sociais pode estar relacionada com o ambiente terapêutico e com o contato com outros pacientes dentro da academia favorecendo a adesão ao treinamento, que no entanto, diferentemente desse estudo, verificou-se um aumento em todos os aspectos avaliados pelo SF-36. Já Benetti et al. ${ }^{25}$ sugere que a melhora nos aspectos sociais, esteja relacionada ao

Tabela 2. Aptidão cardiorrespiratória, porcentagem de massa magra e gorda e teste de repetição máxima pré e pós fase II do programa de reabilitação cardiopulmonar.

\begin{tabular}{|c|c|c|c|}
\hline Variável & Início (média $\pm D P$ ) & Final (média $\pm D P$ ) & $P$ \\
\hline $\mathrm{VO}_{2}$ anaeróbio (ml.kg.min) & $14,5 \pm 3,7$ & $15,8 \pm 4,3$ & $0,00^{*}$ \\
\hline $\mathrm{VO}_{2}$ pico (ml.kg.min) & $22,5 \pm 6,4$ & $23,6 \pm 7,1$ & $0,00^{*}$ \\
\hline $\mathrm{CF} \mathrm{VO}_{2}$ PREDITO & $79,6 \pm 17,8$ & $84,9 \pm 19,3$ & 0,00 * \\
\hline MASSA MAGRA (kg) & $59,8 \pm 9,6$ & $60,7 \pm 9,6$ & $0,00^{*}$ \\
\hline MASSA GORDA (kg) & $24,0 \pm 26,6$ & $17,8 \pm 5,2$ & 0,63 \\
\hline $1 \mathrm{RM}(\mathrm{kg})$ & $71,2 \pm 45,9$ & $100,9 \pm 50$ & 0,00 * \\
\hline
\end{tabular}

$\mathrm{VO}_{2}$ : consumo oxigênio no limiar anaeróbio, $\mathrm{VO}_{2}$ pico: consumo máximo de oxigênio, $\mathrm{VO}_{2}$ predito: consumo máximo de oxigênio predito, 1RM: uma repetição máxima ; valores descritos em média e desvio padrão; *valor de $\mathrm{P}<0,05$ (Teste t pareado). 
maior convívio com a equipe multidisciplinar, informação, entendimento da doença e aderência dos mesmos.

Com relação à força muscular, em um estudo feito por Morais et al. ${ }^{26}$, demonstraram que pacientes submetidos à cirurgia cardíaca têm perda importante de força muscular devido a incisão cirúrgica, dor e tempo de hospitalização. Esta redução de força é revertida com treinamento físico. $\mathrm{Na}$ amostra deste estudo notou-se maior incremento de força nos pacientes submetidos a tratamento clínico, o que reflete a baixa aptidão neuromuscular deste grupo, que mesmo sem ter sido submetido a intervenção conseguiu um melhor aproveitamento do treino de força. Acreditase que tanto os pacientes submetidos ao tratamento cirúrgico como ao clínico melhoraram sua qualidade de vida também pela melhora de sua força muscular, uma vez que a melhora do $\mathrm{VO}_{2}$ pico também depende da melhora da periférica ${ }^{27}$.

Este estudo limita-se pelo número reduzido de prontuários e também pela ausência do grupo controle. Porém é fortalecido pois retrata a realidade clínica de um centro de reabilitação cardíaca o que demonstra o efeito real desse treinamento na qualidade de vida de pacientes portadores de DAC.

\section{CONCLUSÃO}

Conclui-se com este estudo que o programa de treinamento físico contribuiu para a melhora da qualidade de vida, especialmente no que se refere a saúde mental e limitações por aspectos físicos, o que pode ser determinado pela melhora da aptidão cardiorrespiratória e força muscular periférica dos pacientes portadores de DAC.

\section{FONTE DE FINANCIAMENTO}

Nada a declarar.

\section{CONFLITO DE INTERESSES}

Nada a declarar.

\section{REFERÊNCIAS}

1. Andrade JP, Piva e Mattos LA, Carvalho AC, Machado CA, Oliveira GM. National physician qualification program in cardiovascular disease prevention and integral care. Arq Bras Cardiol. 2013 Mar;100(3):203-11. http://dx.doi. org/10.5935/abc.20130061. PMid:23598573.

2. GBD 2017 Causes of Death Collaborators. Global, regional, and national age-sex-specific mortality for 282 causes of death in 195 countries and territories, 1980-2017: a systematic analysis for the Global Burden of Disease Study 2017. Lancet. 2018 Nov 10;392(10159):1736-88. http://dx.doi. org/10.1016/S0140-6736(18)32203-7. PMid:30496103.

3. Christmann M, Costa CC, Moussalle LD. Avaliação da qualidade de vida de pacientes cardiopatas internados. Rev AMRIGS. 2011;55(3):239-43.
4. Santos RD, Timerman S, Spósito S, coordenadores Diretrizes para cardiologistas sobre excesso de peso e doença cardiovascular dos departamentos de aterosclerose, cardiologia clínica e FUNCOR da Sociedade Brasileira de Cardiologia. Arq Bras Cardiol. 2002;78(supl 1):1-14.

5. Brown RA. Rehabilitation of patients with cardiovascular diseases. Report of a WHO expert committee. World Health Organ Tech Rep Ser. 1964;270:3-46. PMid:14128604.

6. Sociedade Brasileira de Cardiologia. Diretriz de Reabilitação Cardíaca. Arq Bras Cardiol. 2005;84(5):431-40. PMid:15917979.

7. Avezum ÁJr, Rassi A Jr, Polanczyk CA, Feitosa GS, Bodanese LC, Herdy AH, et al. Diretriz sul-americana de prevenção e reabilitação cardiovascular. Arq Bras Cardiol. 2014;103(2):131.

8. American College of Sports Medicine. Diretrizes do ACMS para testes de esforço e sua prescrição. 9. ed. Rio de Janeiro: Guanabara Koogan; 2014.

9. Sampaio JKVR, Figueiredo JA No, Queiroz LLC, Sousa RMLD, Reis LMCB, Silva FMAM. Impacto na qualidade de vida pósangioplastia coronariana ou revascularização do miocárdio. Rev Bras Cardiol. 2013;26(5):337-46.

10. McKee $G$. Are there meaningful longitudinal changes in health related quality of life SF36, in cardiac rehabilitation patients? Eur J Cardiovasc Nurs. 2009;8(1):40-7. http://dx.doi. org/10.1016/j.ejcnurse.2008.04.004. PMid:18504159.

11. Favarato MECS, Favarato D, Hueb WA, Aldrighi JM. Qualidade de vida em portadores de doença arterial coronária: comparação entre gêneros. Rev Assoc Med Bras. 2006;52(4):236-41. http://dx.doi.org/10.1590/S010442302006000400023. PMid:16967141.

12. Anderson L, Oldridge $N$, Thompson DR, Zwisler AD, Rees $\mathrm{K}$, Martin N, et al. Exercise-based cardiac rehabilitation for coronary heart disease: cochrane systematic review and meta-analysis. J Am Coll Cardiol. 2016;67(1):1-12. http:// dx.doi.org/10.1016/j.jacc.2015.10.044. PMid:26764059.

13. Hislop H, Avers D, Brown M. Daniels and Worthingham's muscle Testing-E-Book: Techniques of manual examination and performance testing. USA: Elsevier Health Sciences; 2013.

14. Ciconelli RM, Ferraz MB, Santos W, Meinão I, Quaresma MR. Tradução para a língua portuguesa e validação do questionário genérico de Qualidade de Vida SF-36 (Brasil SF-23). Rev Bras Reumatol. 1999;39(3):143-50.

15. Saeidi M, Mostafavi S, Heidari H, Masoudi S. Effects of a comprehensive cardiac rehabilitation program on quality of life in patients with coronary artery disease. ARYA Atheroscler. 2013;9(3):179-85. http://dx.doi.org/10.1136/ heartjnl-2013-304613.387. PMid:23766774.

16. Villelabeitia Jaureguizar K, Vicente-Campos D, Ruiz Bautista L, Hernández de la Peña C, Arriaza Gómez MJ, Calero Rueda $\mathrm{MJ}$, et al. Effect of high-intensity interval versus continuous exercise training on functional capacity and quality of life in patients with coronary artery disease. J Cardiopulm Rehabil Prev. 2016;36(2):96-105. http://dx.doi.org/10.1097/ HCR.0000000000000156. PMid:26872000.

17. Bustamante MJ, Acevedo M, Valentino G, Casasbellas C, Saavedra MJ, Adasme M, et al. Impacto de un programa de rehabilitación cardiovascular fase II sobre la calidad de vida de los pacientes. Ver Chilena Cardiol. 2017;36(1):9-16. http://dx.doi.org/10.4067/S0718-85602017000100001.

18. Takiuti ME, Hueb W, Hiscock SB, Nogueira CR, Girardi $P$, Fernandes F, et al. Quality of life after surgical myocardial revascularization, angioplasty or medical treatment. 
Arq Bras Cardiol. 2007 Maio;88(5):537-44. http://dx.doi. org/10.1590/S0066-782X2007000500007. PMid:17589628.

19. Vidal TMDS, Morais CCA, Carneiro Júnior J, França EÉTD, Batista GR. Implicações da Revascularização do Miocárdio na Qualidade de Vida: três meses de pós-operatório. Rev Bras Ciênc Saúde. 2015;19(3):187-92. http://dx.doi.org/10.4034/ RBCS.2015.19.03.03.

20. Nery RM, Barbisan JN, Mahmud MI. Influence of the practice physical activity in the coronary artery bypass graft surgery results. Rev Bras Cir Cardiovasc. 2007 Jul-Set;22(3):297-302. http://dx.doi.org/10.1590/S0102-76382007000300005. PMid:18157415.

21. Pocock SJ, Henderson RA, Clayton T, Lyman GH, Chamberlain DA. Quality of life after coronary angioplasty or continued medical treatment for angina: three-year follow-up in the RITA-2 trial. Randomized Intervention Treatment of Angina. J Am Coll Cardiol. 2000 Mar 15;35(4):907-14. http://dx.doi. org/10.1016/S0735-1097(99)00637-3. PMid:10732887.

22. Berry JRS, Cunha AB. Avaliação dos efeitos da reabilitação cardíaca em pacientes pós-infarto do miocárdio. Rev Brase Cardiol. 2010;2(23):101-10.

23. Benetti M, Araujo CLP, Santos RZ. Cardiorespiratory fitness and quality of life at different exercise intensities after myocardial infarction. Arq Bras Cardiol. 2010 Set;95(3):399404. http://dx.doi.org/10.1590/S0066-782X2010005000089. PMid:20640381.

24. Anzolin CC, Freire APCFF, Alves MJ, Gomes MJ, Cavalcante MA, Mustafa RM, et al. Treinamento físico em academia melhora qualidade de vida em pacientes no pós-operatório de revascularização miocárdica. ConScientiae Saúde. 2016;30(15):407-13.

25. Benetti M, Nahas MV, Rebelo FP, Lemos LDS, Carvalho TD. Alterações na qualidade de vida de coronariopatas acometidos de infarto agudo de miocárdio, submetidos a diferentes tipos de tratamento. Rev Bras Ativ Fís Saúde. 2001;6(3):27-33.

26. Morais ER, Rassi S, Correa KS, Borges BA. Efeitos do exercício físico não supervisionado na qualidade de vida e na capacidade funcional de portadores de insuficiência cardíaca crônica. J Health Sciences. 2015;15(2):97-103.

27. Gonçalves ACCR, Pastre CM, Camargo JCS Fo, Vanderlei LCM. Exercício resistido no cardiopata: revisão sistemática. Fisioter Mov. 2012;25(1):195-205. http://dx.doi.org/10.1590/ S0103-51502012000100019. 